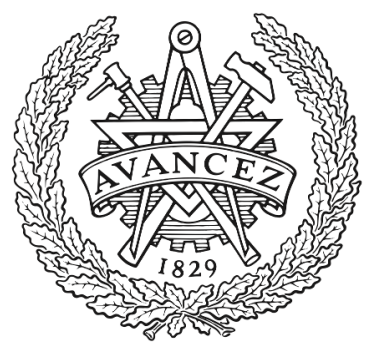

CHALMERS

UNIVERSITY OF TECHNOLOGY

\title{
Creation of Large Quiet Zones in the Presence of Acoustical Levitation Traps
}

Downloaded from: https://research.chalmers.se, 2023-04-26 12:45 UTC

Citation for the original published paper (version of record):

Andersson, C., Ahrens, J. (2021). Creation of Large Quiet Zones in the Presence of Acoustical Levitation Traps. IEEE International Ultrasonics Symposium, IUS.

http://dx.doi.org/10.1109/IUS52206.2021.9593601

N.B. When citing this work, cite the original published paper. 


\title{
Creation of Large Quiet Zones in the Presence of Acoustical Levitation Traps
}

\author{
Carl Andersson, Jens Ahrens \\ Division of Applied Acoustics, Chalmers University of Technology, Gothenburg, Sweden \\ $\{$ carl.andersson, jens.ahrens\}@chalmers.se
}

\begin{abstract}
We propose a method to generate an acoustical levitation trap at the same time as suppressing the sound in a multi-wavelength region of space. The method uses a spherical basis expansion of the sound field in the quiet zone, calculated by translating individual source expansions of elements in a transducer array. We show that it is possible to control the size of the quiet zone with the truncation order of the expansion, and explain the trade-off between field suppression in the quiet zone and stiffness loss of the levitation trap. Measurements of a generated sound field show the existence of a region of lower sound pressure. Simulations demonstrate a contrast up to $50 \mathrm{~dB}$ and sizes up to $60 \mathrm{~mm}$ for a 256 element array.
\end{abstract}

Index Terms-Acoustic Levitation, Transducer Arrays, Sound Field Control

\section{INTRODUCTION}

Acoustic levitation is a useful technique for non-contact manipulation of small lightweight objects. In most cases, the levitation is done in free space, so that one or more levitation traps can be designed without considering other objects in the sound field $[1,2]$. This is a feasible approach when there are no external objects in the vicinity of the target levitation object, and the traps for all target objects are designed together. However, the free field assumption fails if there are scattering objects present in the sound field. These scattering objects can be e.g. required parts of the hardware setup, external tooling, or display elements [3]. We proposed earlier to use superposition of sound fields with mutual quiet zones and levitation traps to levitate multiple objects, in which case the additional scattering objects are the other levitating objects [4, 5]. Work has been presented by other authors on creating self-bending beams with physical acoustical lenses to avoid scatterers that are close to the array [6]. One major disadvantage of this acoustic lens approach is that the lens is static, preventing reconfiguration of the levitation system during operation.

One intuitive way to avoid unwanted scattering from external objects in the sound field is to create a region of space around the object where the sound pressure is low enough that the scattering can be disregarded. This is preferable to full modeling of the scattering from the object due to computational complexity of such modeling, as well as errors stemming from model inaccuracies. We propose a way to create large quiet zones to avoid arbitrary scatterers in the sound field.

\section{METHOD}

Similar to our earlier work on simultaneous levitation of multiple objects using quiet zones, our proposed method is based on optimizing the driving phases and magnitudes of transducer elements in an array to minimize a cost function [4]. This cost function consists of two parts, one which describes the levitation trap for the desired levitation object, and one which describe the quiet zone. The choice of trap description is in principle independent from the quiet zone description, as long as it can be represented as a single-value real cost. In this paper we choose to target small lightweight objects, due to the simplicity of the cost function and wide spread use of such methods [7, 8].

The quiet zone part of the cost function should represent the overall quietness in the desired region. One way to describe this would be to sample the sound pressure at strategically chosen points in the region, and design a cost function from those discrete points. However, such a sampling would have to be dense enough so that the sound field cannot have substantially different magnitude in between the sample points and at the sample points. To avoid a dense sampling of the quiet region we instead choose to represent the sound field as a spherical basis expansion around the center of the desired quiet region [9]. While an infinite expansion is needed to fully represent the sound field, similar to a Taylor expansion, a truncated expansion is sufficient to accurately describe the sound field within a certain expansion radius [10]. Thus, the sound field $p$ at a point $\vec{r}$ close to the center of the desired quiet zone $\vec{r}_{q}$ is described as

$$
p(\vec{r})=\sum_{n=0}^{N} \sum_{m=-n}^{n} S_{n}^{m}\left(\vec{r}_{q}\right) j_{n}(k \hat{r}) Y_{n}^{m}(\hat{\theta}, \hat{\phi})
$$

where $(\hat{r}, \hat{\theta}, \hat{\phi})$ are the spherical coordinates of the point $\vec{r}$ relative to the center or the quiet zone, $j_{n}$ are spherical Bessel functions, $Y_{n}^{m}$ are spherical harmonics, $k=\omega / c$ is the wavenumber of the sound field, $S_{n}^{m}\left(\vec{r}_{q}\right)$ are the local expansion coefficients of the sound field, and $N$ is the truncation order for the expansion.

Since the sound field in question is generated by a transducer array, it can be described as the superposition of the sound fields generated by the individual transducers. Expanding the sound field in the spherical basis functions is a linear operation, thus the expansion coefficients of the total sound field are the superposition of the expansion coefficients of the 
fields generated by all the individual transducers. This can be written in terms of normalized expansions as

$$
S_{n}^{m}\left(\vec{r}_{q}\right)=\sum_{l} Q_{l} S_{n, l}^{m}\left(\vec{r}_{q}\right)
$$

where $Q_{l}$ is the complex driving amplitude for transducer $l$, and $S_{n, l}^{m}\left(\vec{r}_{q}\right)$ is the local expansion of the field generated by said transducer when driven at unit magnitude and reference phase. The local expansions for all the individual transducers are obtained by applying translation theorems for spherical wave expansions to a single expansion representing the source behavior. The details of translation theorems for spherical wave expansions is too involved to cover in this paper, and we refer the interested reader to other literature [10]. The source expansion can stem from analytical derivations of the source characteristics, or from fitting an expansion to measured data. In this paper we used measured data of a single transducer located in the central portion of the array, and performed a least squares fit of the expansion coefficients. Since the transducer geometry is rotationally symmetric, the fit was performed under the assumption that the source radiation is also rotationally symmetric. This fit produced a mean absolute relative error of roughly one decibel when used to recompute the sound field at the measured positions.

It is easy to see from (1) that minimizing the magnitude of all the expansion coefficients $S_{n}^{m}\left(\vec{r}_{q}\right)$ will also minimize the overall magnitude of the sound field inside the expansion radius. The summed square magnitudes of the expansion coefficients is therefore a basic single value descriptor of the quietness in the quiet zone. However, the expansion coefficients are not of the same typical magnitude, which causes the minimization of the summed square magnitudes to prioritize certain coefficients over others. To counteract this imbalance, the actual expansion coefficients are pre-scaled with typical values before the reduction to a single quantifying value. These typical values are calculated using the spherical Hankel functions $h_{n}$, as

$$
M_{n}=Q_{\max } k\left|h_{n}(k \tilde{r})\right| \sqrt{4 \pi(2 n+1)},
$$

i.e. the magnitude of monopole source expansion coefficients evaluated at the mean distance between the transducers in the array and the center of the quiet zone, $\tilde{r}$, scaled by the maximum amplitude $Q_{\max }$ of the transducer elements.

The cost function can finally be expressed as

$$
O\left(\left\{Q_{l}\right\}\right)=O_{t}\left(\left\{Q_{l}\right\} ; \vec{r}_{t}\right)+w \sum_{n, m}^{n \leq N}\left|\sum_{l} Q_{l} \frac{S_{n, l}^{m}\left(\vec{r}_{q}\right)}{M_{n}}\right|^{2}
$$

where $O_{t}$ is a cost function for a trap, evaluated at the target trap location $\vec{r}_{t}$, and $w$ is a weight controlling the relative importance of the quiet zone and the trap. For simplicity, we choose to reuse an earlier trap cost function proven to create stable levitation traps [11, Eq. (5)]. By changing the truncation order $N$ for the sound field expansion, the region considered for the quiet zone can be controlled, where a higher truncation order considers a larger region. This cost function is minimized using a Broyden-Fletcher-Goldfarb-Shanno

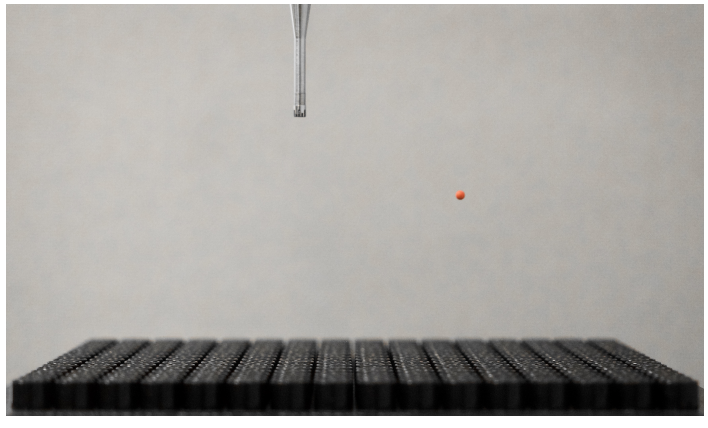

Fig. 1. Photograph of the array, a levitating red polystyrene ball, and the measurement microphone in the quiet zone.

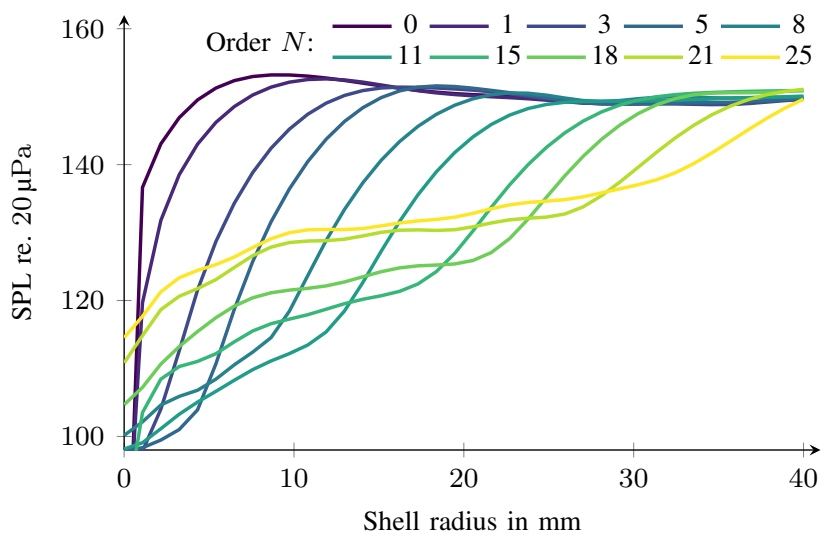

Fig. 2. Mean sound pressure level on concentric spherical shells of varying radius in the quiet zone for different orders of the quiet zone.

(BFGS) style algorithm, with the phases and magnitudes of the transducer amplitudes as the variables [12, 13]. Since the cost function (2) is non-convex, it is relevant to initialize the optimization at a favorable state. We therefore choose to start each minimization at phases and magnitudes which are known to create a levitation trap at the trap position, i.e. a minimum of the trap cost function $O_{t}$. The quiet zone is thereafter included one order at the time, each optimization stage starting from the solution of the previous. Since the cost function only changes slightly with each new order, this sequential optimization will track local minima through the non-convex solution space.

\section{RESULTS}

The proposed method for quiet zone and levitation trap generation was applied to an example setup, chosen to match hardware setups used in many experiments. This consist of a 16x16 planar transducer array operating at $40 \mathrm{kHz}$, with transducers spaced in a square grid with an element spacing of $10.5 \mathrm{~mm}$. Note that the element spacing is larger that one wavelength, which indicates that there is spatial aliasing in the sound field, i.e. the array does not have optimal ability to control the sound field. The trap and the quiet zone was placed $50 \mathrm{~mm}$ and $67 \mathrm{~mm}$ above the array respectively, the trap $30 \mathrm{~mm}$ to one side and the quiet zone $15 \mathrm{~mm}$ to the other, relative to the center of the array, see Fig. 1. 


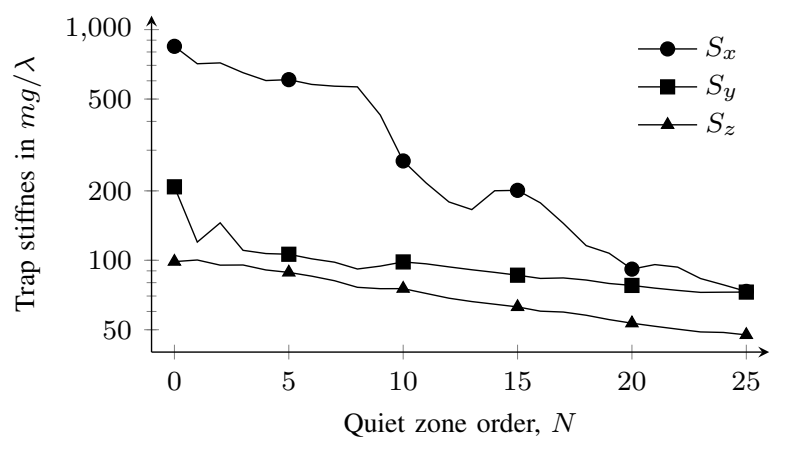

Fig. 3. Trap stiffness for varying quiet zone order.

The performance in the quiet zone was evaluated by calculating the mean sound pressure level on concentric spherical shells centered at the target quiet zone location. As seen in Fig. 2, the sound pressure level is indeed lower in the center of the quiet zone compared to outside the zone. It is also clear that the zone order $N$ has the intended effect of changing the overall size of the zone, where a higher zone order creates a larger region where the sound pressure is suppressed.

The performance of the trap is shown in Fig. 3, and is evaluated as the trap stiffness in the axial directions of the coordinate system. It can be seen that the trap stiffness is reduced when the quiet zone order is increased, i.e. the trap is weaker when the quiet zone is larger. However, this reduction of stiffness is not a significant issue since the forces around the trap are several times stronger than the gravitational pull on the object.

A slice of the sound field generated by the array around a quiet zone of order 15 can be seen in Fig. 4 (simulated) and Fig. 5 (measured). Both of these indicate the existence of a quiet zone of similar qualitative shape and sound pressure, also in line with Fig. 2. However, the high pressure region to the left in the investigated slice has a lower sound pressure level in measurements than in simulations. This could be caused by an overestimation of the strength of the transducer elements used in modeling, and effectively decreases the contrast between quiet zone and the rest of the field when comparing the measured field and the simulated field.

\section{Discussion}

When the two evaluations in Fig. 2 and Fig. 3 are considered together, it is clear that the creation of a quiet zone has an influence on the levitation trap. It can also be seen in Fig. 2 that the pressure level in the interior of the quiet zone is higher for high zone orders compared to low orders, even though the trap is weakened. This is likely due to the circumstance that the the overall objective is more demanding, and it is not possible to maintain the same trap strength, quiet zone size, and quiet zone strength. In essence there is a trade off between trap stiffness and the size and strength of the quiet zone, controlled by the zone order $N$ and the quiet zone weight $w$, see (2). If the quiet zone weight is increased the suppression in the quiet zone is enhanced for higher orders as well, but at the expense

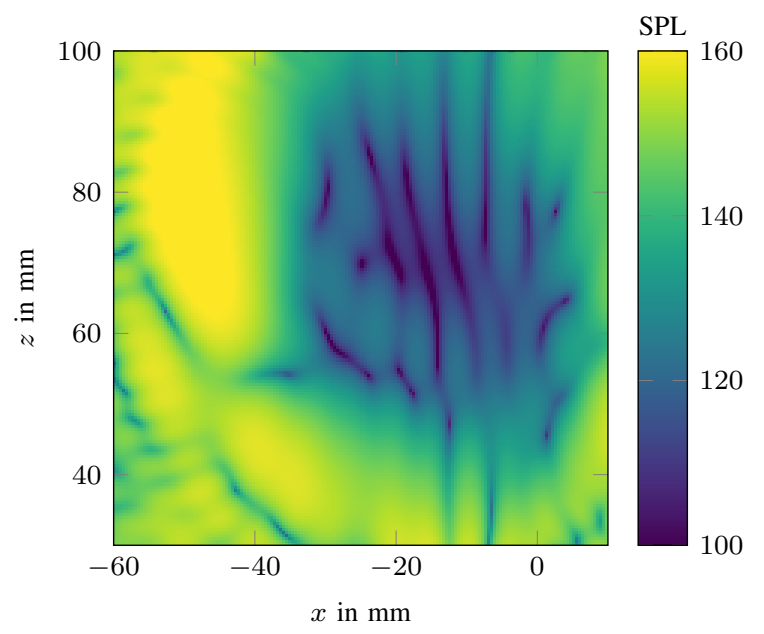

Fig. 4. Simulated sound field around the quiet zone.

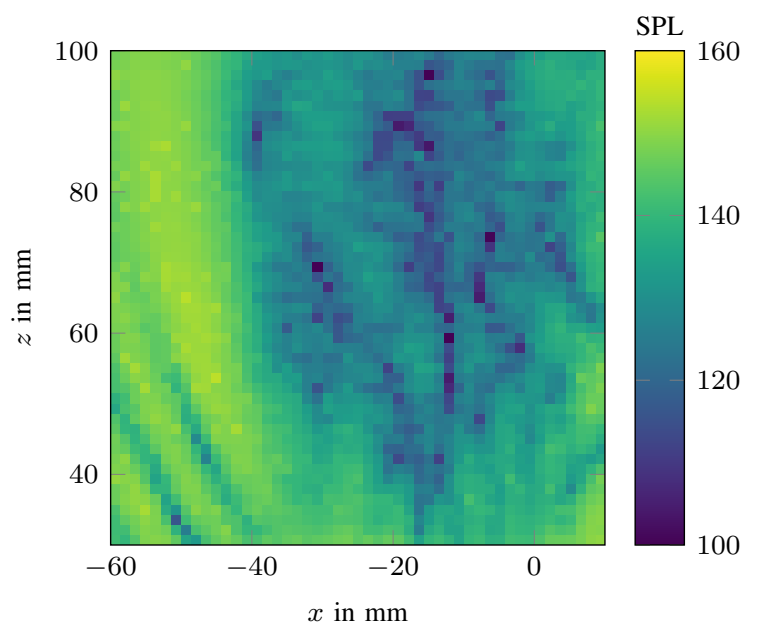

Fig. 5. Measured sound field around the quiet zone.

of the stiffness of the trap. This indicates that there is a limit on how large the quiet zone can become before either the trap or the quiet zone breaks down. For the investigated geometry, the trap and the center of the quiet zone are approximately $48 \mathrm{~mm}$ apart. It is easy to understand that the quiet zone cannot include the region too close to the trap and still be successful, since the trap requires high sound pressures to exist.

The difference between the measured and simulated sound fields seen in Figs. 4 and 5 has two likely causes: the directivity model and the array calibration. Our modeling assumes that all transducers have the same radiation characteristic, obtained from measuring a single transducer in the array. It is likely that there is some variation of the transducer behavior, in particular between elements in the center of the array and those along the edges. The array has transducers arranged in a square grid which could introduce an azimuth dependency in the transducer directivity, which would not show in our measurements of the transducer directivity and the resulting fit of the source expansion. Secondly, the elements in the specific hardware array used to perform the measurement of the sound field are only phase calibrated, not amplitude calibrated. This 
means that some of the elements could be stronger or weaker than assumed. A quiet zone is formed by mutual cancellation of the sound fields from the transducers, and is thus more sensitive to errors in the magnitude of each of the individual fields, compared to e.g. the strength of a focus point or a levitation trap. That there is still a quiet zone in the measured field indicates that this approach is somewhat resilient to array mis-match, which is preferable to approaches that require a full characterization of each element in the array.

\section{CONCLUSION}

We have presented a method for generating a levitation trap and at the same time suppressing the sound field in a different, but nearby, region of space. Our experiments and simulations indicate that the approach is reasonably effective, achieving a sound pressure contrast of up to $50 \mathrm{~dB}$ for small regions, and about $30 \mathrm{~dB}$ for regions of a diameter of approximately $40 \mathrm{~mm}$. While this is achieved at the expense of the trap stiffness, it is still strong enough to levitate the target object in the simulated cases as well as in the single experimentally tested case. For heavier objects, it is possible to modify the weights used in the numerical optimization to prioritize the trap at the expense of a weaker quiet zone.

This method could be used to levitate multiple large objects by superposing multiple sound fields with mutual traps and quiet zones. This requires that a suitable cost function for single large object levitation is used, as well as hardware with sufficient sound power output and spatial control of the sound field. If the levitated objects are close in proximity to each other there can be multiple scattering occurring between the objects, which is not directly handled by this approach. However, this could be handled by including the scattering in the sound field model for the quiet zone. The steps required to do so are not difficult in principle, but tedious in implementation.

\section{REFERENCES}

[1] Diego Martinez Plasencia et al. "GS-PAT: High-Speed Multi-Point Sound-Fields for Phased Arrays of Transducers". en. In: ACM Transactions on Graphics 39.4 (July 2020). ISSN: 0730-0301, 1557-7368. DOI: 10 . 1145/3386569.3392492.

[2] Asier Marzo and Bruce W. Drinkwater. "Holographic Acoustic Tweezers". en. In: Proceedings of the National Academy of Sciences 116.1 (Jan. 2019), pp. 84-89. ISSN: 0027-8424, 1091-6490. DOI: 10.1073 / pnas . 1813047115.

[3] Rafael Morales et al. "LeviProps: Animating Levitated Optimized Fabric Structures Using Holographic Acoustic Tweezers". en. In: Proceedings of the 32nd Annual ACM Symposium on User Interface Software and Technology. New Orleans LA USA: ACM, Oct. 2019, pp. 651-661. ISBN: 978-1-4503-6816-2. DOI: 10. $1145 / 3332165.3347882$.
[4] Carl Andersson and Jens Ahrens. "A Method for Simultaneous Creation of an Acoustic Trap and a Quiet Zone". In: 2018 IEEE 10th Sensor Array and Multichannel Signal Processing Workshop (SAM). IEEE, July 2018, pp. 622-626. ISBN: 978-1-5386-4752-3. DOI: 10. 1109/SAM.2018.8448949.

[5] Carl Andersson and Jens Ahrens. "Minimum Trap Separation for Acoustical Levitation Using Phased Ultrasonic Transducer Arrays". en. In: Proceedings of the 23rd International Congress on Acoustics. Aachen, Germany, Sept. 2019, pp. 1117-1123. ISBN: 978-3939296-15-7.

[6] Mohd Adili Norasikin et al. "SoundBender: Dynamic Acoustic Control Behind Obstacles". In: Proceedings of the 31st Annual ACM Symposium on User Interface Software and Technology. UIST '18. New York, NY, USA: Association for Computing Machinery, Oct. 2018, pp. 247-259. ISBN: 978-1-4503-5948-1. DOI: 10.1145/ 3242587.3242590.

[7] Asier Marzo et al. "Holographic Acoustic Elements for Manipulation of Levitated Objects". In: Nature Communications 6.1 (Oct. 2015), p. 4316. DOI: 10 . 1038/ncomms9661.

[8] Julie R Williamson, Euan Freeman, and Stephen Brewster. "Levitate: Interaction with Floating Particle Displays". In: Proceedings of the 6th ACM International Symposium on Pervasive Displays - PerDis '17. New York, New York, USA: ACM, June 2017. ISBN: 978-14503-5045-7. DOI: 10.1145/3078810.3084347.

[9] Earl G Williams. Fourier Acoustics. Sound Radiation and Nearfield Acoustical Holography. Elsevier, June 1999. ISBN: 978-0-08-050690-6.

[10] N A Gumerov and R Duraiswami. Fast Multipole Methods for the Helmholtz Equation in Three Dimensions. Elsevier, 2005. ISBN: 978-0-08-044371-3.

[11] Carl Andersson and Jens Ahrens. "Reducing Spiraling in Transducer Array Based Acoustic Levitation". In: 2020 IEEE International Ultrasonics Symposium (IUS). Las Vegas, NV, USA: IEEE, Sept. 2020, pp. 1-4. ISBN: 978-1-72815-448-0. DOI: 10 . 1109 / IUS46767 . 2020 . 9251489.

[12] Ciyou Zhu et al. "Algorithm 778: L-BFGS-B: Fortran Subroutines for Large-Scale Bound-Constrained Optimization". In: ACM Transactions on Mathematical Software (TOMS) 23.4 (Dec. 1997), pp. 550-560. DOI: 10.1145/279232.279236.

[13] Pauli Virtanen et al. "SciPy 1.0: Fundamental Algorithms for Scientific Computing in Python". en. In: Nature Methods (Feb. 2020), pp. 1-12. ISSN: 15487105. DOI: 10.1038/s41592-019-0686-2. 